\title{
Analytical and numerical study of the electro-osmotic annular flow of viscoelastic fluids
}

\author{
L.L. Ferrás $^{\text {a,* }}{ }^{\text {, A.M. Afonso }}{ }^{\text {b }}$, M.A. Alves ${ }^{b}$, J.M. Nóbrega ${ }^{a}$, F.T. Pinho ${ }^{c}$ \\ ${ }^{a}$ Institute for Polymers and Composites/I3N, University of Minho, Campus de Azurém, 4800-058 Guimarães, Portugal \\ ${ }^{\mathrm{b}}$ Departamento de Engenharia Química, CEFT, Faculdade de Engenharia da Universidade do Porto, Rua Dr. Roberto Frias s/n, $4200-465$ Porto, Portugal \\ ${ }^{c}$ Centro de Estudos de Fenómenos de Transporte, Faculdade de Engenharia da Universidade do Porto, Rua Dr. Roberto Frias s/n, 4200-465 Porto, Portugal
}

\section{A R T I C L E I N F O}

\section{Article history:}

Received 19 September 2013

Accepted 15 December 2013

Available online 26 December 2013

\section{Keywords:}

Annular flow

Analytical solution

sPTT model

Slip boundary conditions

\begin{abstract}
A B S T R A C T
In this work we present semi-analytical solutions for the electro-osmotic annular flow of viscoelastic fluids modeled by the Linear and Exponential PTT models. The viscoelastic fluid flows in the axial direction between two concentric cylinders under the combined influences of electrokinetic and pressure forcings. The analysis invokes the Debye-Hückel approximation and includes the limit case of pure electro-osmotic flow. The solution is valid for both no slip and slip velocity at the walls and the chosen slip boundary condition is the linear Navier slip velocity model. The combined effects of fluid rheology, electro-osmotic and pressure gradient forcings on the fluid velocity distribution are also discussed.
\end{abstract}

(c) 2013 Elsevier Inc. All rights reserved.

\section{Introduction}

With the evolution of diagnostic tools that capture flow characteristics at the microscale there has been growing evidence of wall slip in experiments using both Newtonian and non-Newtonian fluids [1]. Simultaneously, molecular dynamics has also helped question the veracity of the no-slip law and, nowadays, the wall slip velocity phenomenon in some fluid flows is accepted [2], especially for viscoelastic fluids. In particular, the works of Denn [1] and Lauga et al. [2] provide insights on what has already been done regarding slip velocity measurements and theoretical approaches for Newtonian and non-Newtonian fluids. Kazatchkov and Hatzikiriakos [3] and Hatzikiriakos [4] provide novel physical models that are able to capture the slippery characteristics of certain viscoelastic fluids. All these works concern pressure-driven flows.

For electro-osmotic driven flows the existence of wall slip has been more readily accepted. When an electrolyte solution flows in channels made from dielectric materials, a thin electric double layer (EDL) is spontaneously formed in the vicinity of the wall, where the imbalance of positive and negative ions can be used by an applied electric potential to induce flow along the channel. This layer is usually very small in such a way that the bulk flow can be modeled accurately considering the linear Navier [5] slip boundary condition at the wall [6-11].

\footnotetext{
* Corresponding author.

E-mail addresses: luis.ferras@dep.uminho.pt (L.L. Ferrás), aafonso@fe.up.pt (A.M. Afonso), mmmalves@fe.up.pt (M.A. Alves), mnobrega@dep.uminho.pt (J.M. Nóbrega), fpinho@fe.up.pt (F.T. Pinho).
}

In order to ascertain whether the proposed slip models are reliable, analytical solutions and numerical simulations are important tools. Additionally, the analytical solutions can be of major importance in the verification of numerical codes. These two facts, together with the urge of understanding the electro-osmotic flow and the slip phenomenon typical in viscoelastic fluid flows, are the main motives for this work. On what concerns analytical solutions for viscoelastic fluids with slip boundary conditions, we can distinguish two cases: pressure-driven viscoelastic fluid motion; viscoelastic fluid motion driven by a combination of electro-osmotic and pressure forcings.

For Newtonian fluids, Ngoma and Erchiqui [12] investigated numerically the effects of heat flux and boundary slip on electrokinetic flows. Soong et al. [11] analyzed pressure-driven electrokinetic flows in hydrophobic microchannels with emphasis on the slip effects under coupling of interfacial electric and fluid slippage phenomena while Jamaati et al. [13] studied the pressure-driven electrokinetic slip-flow in planar microchannels. For non-Newtonian fluids only analytical solutions under no-slip boundary conditions could be found. Zhao and Yang [14] reported a theoretical analysis of electro-osmotic mobility of non-Newtonian fluids and Afonso et al. [15,16] presented an analytical solution for the mixed electro-osmotic/pressure driven flow of viscoelastic fluids in microchannels and for the case of electro-osmotic flow under symmetric and asymmetric zeta potential, respectively. All these analytical solutions were derived for simple channel flows.

For an annular geometry the literature is rich in analytical solutions for the pressure driven case [17-22] with applications to the oil and gas industries. For the electro-osmotic flow through an annulus, the applications to real life are becoming important in 
biological systems as in electrophoretic separation of proteins and for blending chemical and biological fluids [26]. Regarding analytical studies of such flows we could only find the works of Tsao and Kang et al. $[23,24]$ where the electroosmotic flow of a Newtonian fluid through an annulus was studied for high and low zeta potentials, the work of Goswami and Chakraborty [25] where the authors present semi-analytical solutions for electroosmotic flows of Newtonian fluids with interfacial slip in microchannels of complex cross-sectional shape, including an annular geometry, Jian et al. [26] who analyzed the behavior of time periodic electroosmosis in a cylindrical microannulus, and more recently, Shamshiri et al. [27], who studied electroviscous and thermal effects on the electro-osmotic flow of power-law fluids through an annulus. Analytical solutions for the viscoelastic annular flow case under the influence of both electro-osmotic and pressure driven forcings could not be found in the literature. Given this limitation, in this work we present a semi-analytical solution for the pure axial flow of the Linear and Exponential PTT models [28,29] that is valid for both no-slip and slip boundary conditions.

Although this flow is known to be of interest for industry, we could not find any experimental data. Therefore, we derived a general solution that can cope with various degrees of slip and different classes of fluids. Also, the solution can be easily adapted to other viscoelastic models.

The remaining of this paper presents the relevant set of governing equations, followed by their solutions. A discussion of the effects of the various relevant dimensionless parameters upon the flow characteristics closes this work.

\section{Governing equations}

The flow of interest is governed by the continuity equation,

$\nabla \cdot \mathbf{u}=0$

and by the general Cauchy momentum equation,

$\rho \frac{\partial \mathbf{u}}{\partial t}+\rho \nabla \cdot \mathbf{u} \mathbf{u}=-\nabla p+\nabla \cdot \tau+\rho_{e} \mathbf{E}$

where $\mathbf{u}$ is the velocity vector, $p$ is the pressure, $\rho$ is the density and $\rho_{e} \mathbf{E}$ represents the electrical force per unit volume acting upon the ions in fluid. This force depends on $\mathbf{E}$, the applied external electric field, and on $\rho_{e}$, the net electric charge density. This charge density distribution is a consequence of the distribution of the spontaneously formed electric double layers, which are assumed here not to be affected by the imposed electric field. The deviatoric stress tensor, $\tau$, describes the fluid rheological behavior here given by the simplified Phan-Thien-Tanner (sPTT) model $[28,29]$,

$$
\begin{aligned}
& f(\operatorname{tr} \tau) \tau+\lambda\left(\frac{\partial \tau}{\partial t}+\mathbf{u} \cdot \nabla \tau-\left[(\nabla \mathbf{u})^{T} \cdot \tau+\tau \cdot \nabla \mathbf{u}\right]\right) \\
& \quad=\eta\left(\nabla \mathbf{u}+(\nabla \mathbf{u})^{T}\right)
\end{aligned}
$$

where $\eta$ is the polymer viscosity coefficient, $\lambda$ is the relaxation time and $f(\operatorname{tr} \tau)$ is a function depending on the trace of the stress tensor specifying the various versions of this class of models [28,29],

$f(\operatorname{tr} \tau)= \begin{cases}1+\frac{\varepsilon \lambda}{\eta} \tau_{k k} & \text { linear } \\ \exp \left(\frac{\varepsilon \lambda}{\eta} \tau_{k k}\right) & \text { exponential }\end{cases}$

As for the boundary conditions, the no-slip boundary condition at the wall is expressed as $\mathbf{u}=\mathbf{0}$, whereas the linear Navier slip law [5], is given by

$u_{\text {slip }}= \pm \mathcal{L} \tau_{r z}$

where $\mathcal{L}$ is the slip coefficient and $\tau_{r z}$ is the wall shear stress. For the inner cylinder wall the plus sign is considered, while for the outer cylinder wall the minus sign is used.

\section{Semi-analytical solution}

We assume the flow between the two concentric cylinders is fully developed, with the streamwise velocity component in the $z$ direction (the direction of the axes of the cylinders) only depending on the radial coordinate, $r$. As shown in Fig. 1 the outer cylinder has a radius $R$, and the radius of the inner cylinder is given by $\alpha R$ with $0<\alpha<1$. The gap between the two cylinders is $\delta=R(1-\alpha)$. We further assume that there is no rotation, that the flow is axisymmetric and it is fully developed. For such conditions, continuity, momentum and the constitutive equations can be further simplified.

The axial momentum equation in cylindrical coordinates is given by,

$\frac{1}{r} \frac{\mathrm{d}\left(r \tau_{r z}\right)}{\mathrm{d} r}=-\rho_{e} E_{z}+p_{, z}$

where $p_{z}$ is the constant pressure gradient in the $z$ direction, $\tau_{r z}$ is the non-zero shear stress and $E_{z} \equiv-\mathrm{d} \Phi / \mathrm{d} z$ with $\Phi=\psi+\phi$, where $\phi$ is the applied streamwise potential and $\psi$ is the equilibrium/induced potential across the cylinders' gap, associated with the interaction between the ions of the fluid and the dielectric wall. The charge density, $\rho_{e}$, is related to the electric potential by $\rho_{e}=-\epsilon \kappa^{2} \psi(r)$ assuming the Debye-Hückel approximation (at room temperature this limits the potential at the wall to values much smaller than $26 \mathrm{mV}$ ), and the induced electric field is given by the solution of the following differential equation [23],

$\frac{1}{r} \frac{\mathrm{d}}{\mathrm{d} r}\left(r \frac{\mathrm{d} \psi}{\mathrm{d} r}\right)=\kappa^{2} \psi$

where $\kappa^{2}$ is the Debye-Hückel parameter. For the boundary conditions $\psi(\alpha R)=\zeta_{i}$ and $\psi(R)=\zeta_{0}$, the solution of Eq. (7) is given by [23],

$\psi(r)=\frac{I_{0}(\kappa r)\left[\zeta_{0} K_{0}(\alpha \kappa R)-\zeta_{i} K_{0}(\kappa R)\right]+K_{0}(\kappa r)\left[\zeta_{i} I_{0}(\kappa R)-\zeta_{0} I_{0}(\alpha \kappa R)\right]}{I_{0}(\kappa R) K_{0}(\alpha \kappa R)-I_{0}(\alpha \kappa R) K_{0}(\kappa R)}$

where $I_{0}($.$) and K_{0}($.$) are the modified Bessel functions of first and$ second kind, respectively.

The induced electric potential is now easily computed by $\rho_{e}=-\epsilon \kappa^{2} \psi(r)$ with $\psi(r)$ given by Eq. (8), where $\epsilon$ is the dielectric constant of the fluid.

Integration of Eq. (6) results in the following expression for the shear stress,

$$
\begin{aligned}
\tau_{r z}= & \frac{r p_{, z}}{2}+\epsilon \kappa E_{z} \frac{K_{1}(\kappa r)\left[-\zeta_{i} I_{0}(\kappa R)+\zeta_{0} I_{0}(\kappa \alpha R)\right]}{I_{0}(\kappa R) K_{0}(\alpha \kappa R)-I_{0}(\alpha \kappa R) K_{0}(\kappa R)}+\frac{\epsilon \kappa^{2} E_{z} r}{2} \\
& \times \frac{{ }_{0} F_{1}\left[2,\left(\frac{\kappa r}{2}\right)^{2}\right]\left[-\zeta_{i} K_{0}(\kappa R)+\zeta_{0} K_{0}(\kappa \alpha R)\right]}{I_{0}(\kappa R) K_{0}(\alpha \kappa R)-I_{0}(\alpha \kappa R) K_{0}(\kappa R)}+\frac{c_{1}}{r}
\end{aligned}
$$

where ${ }_{0} F_{1}\left(2,\left(\frac{\kappa r}{2}\right)^{2}\right)$ is the confluent hypergeometric limit function, which is defined as $[30,31]$

${ }_{0} F_{1}(2, z)=\sum_{k=0}^{\infty} \frac{z^{k}}{k !(k+1) !}$

and $c_{1}$ is the constant of integration that can be determined assuming that $\tau_{r z}=0$ for $r=\beta R$ with $\alpha<\beta<1$,

$$
\begin{aligned}
c_{1}= & -\frac{(\beta R)^{2} p_{, z}}{2}-\epsilon \kappa E_{z} \beta R \frac{K_{1}(\kappa \beta R)\left(-\zeta_{i} I_{0}(\kappa R)+\zeta_{0} I_{0}(\kappa \alpha R)\right)}{I_{0}(\kappa R) K_{0}(\alpha \kappa R)-I_{0}(\alpha \kappa R) K_{0}(\kappa R)} \\
& -\frac{\epsilon \kappa^{2} E_{z}(\beta R)^{2}}{2} \frac{{ }_{0} F_{1}\left[2,\left(\frac{\kappa \beta R}{2}\right)^{2}\right]\left(-\zeta_{i} K_{0}(\kappa R)+\zeta_{0} K_{0}(\kappa \alpha R)\right)}{I_{0}(\kappa R) K_{0}(\alpha \kappa R)-I_{0}(\alpha \kappa R) K_{0}(\kappa R)}
\end{aligned}
$$

This implies that $\beta$ has to be determined as part of the solution.

For the pure axial annular flow the shear stress component is given by,

$f\left(\tau_{z z}\right) \tau_{r z}=\eta \frac{\mathrm{d} u}{\mathrm{~d} r}$ 

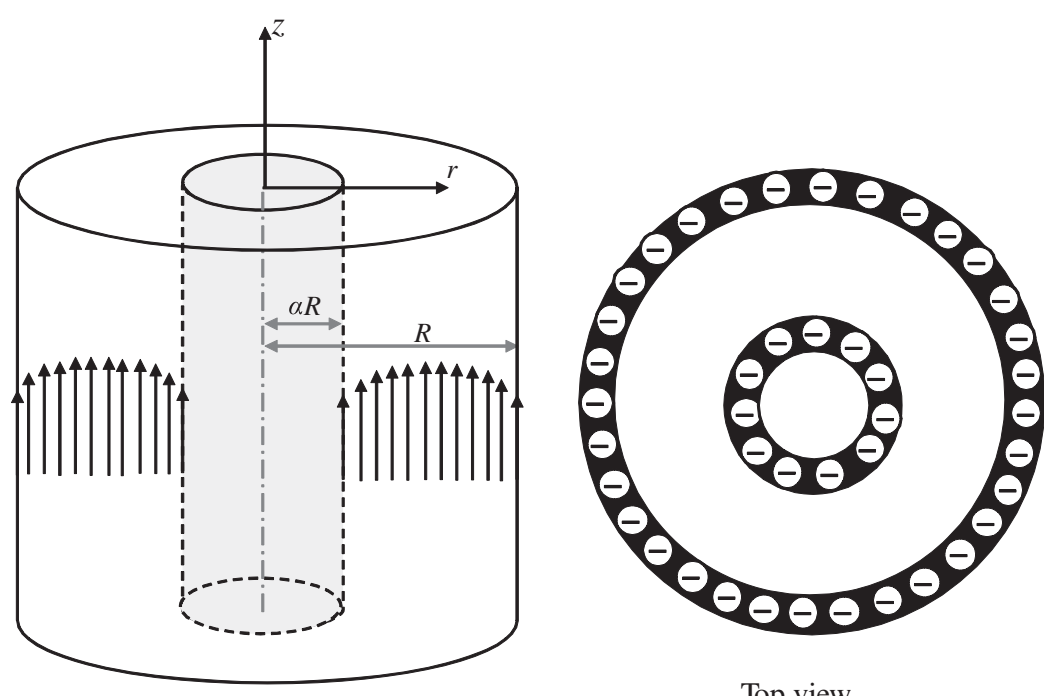

Top view

Fig. 1. Schematic of the electro-osmotic annular slip flow.

and the normal stress distribution can be determined once the shear component is known using

$f\left(\tau_{z z}\right) \tau_{z z}=2 \lambda \tau_{r z} \frac{\mathrm{d} u}{\mathrm{~d} r}$

which leads to

$\tau_{z z}=\frac{2 \lambda}{\eta} \tau_{r z}^{2}$

since $\tau_{r r}=0$. More details are given in the work of Pinho and Oliveira [20].

\subsection{Linear and Exponential PTT models}

Combining Eqs. (12) and (14), the velocity gradient for the Linear and Exponential PTT models can be written as,

$\frac{\mathrm{d} u}{\mathrm{~d} r}=\frac{\tau_{r z}}{\eta}+\frac{2 \varepsilon \lambda^{2}}{\eta^{3}} \tau_{r z}^{3}$

and

$\frac{\mathrm{d} u}{\mathrm{~d} r}=\frac{\tau_{r z}}{\eta} \exp \left(\frac{2 \varepsilon \lambda^{2}}{\eta^{2}} \tau_{r z}^{2}\right)$

respectively.

$$
\begin{aligned}
\tau_{r z}(r)= & \frac{\left[r-\frac{(\beta R)^{2}}{r}\right] p_{, z}}{2} \\
& +\epsilon \kappa E_{z} \frac{\left(-\zeta_{i} I_{0}(\kappa R)+\zeta_{0} I_{0}(\kappa \alpha R)\right)}{I_{0}(\kappa R) K_{0}(\alpha \kappa R)-I_{0}(\alpha \kappa R) K_{0}(\kappa R)}\left[K_{1}(\kappa r)-\frac{\beta R}{r} K_{1}(\kappa \beta R)\right] \\
& +\frac{\epsilon \kappa^{2} E_{z}}{2} \frac{-\zeta_{i} K_{0}(\kappa R)+\zeta_{0} K_{0}(\kappa \alpha R)}{I_{0}(\kappa R) K_{0}(\alpha \kappa R)-I_{0}(\alpha \kappa R) K_{0}(\kappa R)} \\
& \times\left\{r_{0} F_{1}\left[2,\left(\frac{\kappa r}{2}\right)^{2}\right]-\left[\frac{(\beta R)^{2}}{r}\right]{ }_{0} F_{1}\left[2,\left(\frac{\kappa \beta R}{2}\right)^{2}\right]\right\}
\end{aligned}
$$

\subsubsection{Linear PTT model}

Upon integration of Eq. (15) and using the boundary condition $u(\alpha R)$, for the Linear viscoelastic model we obtain the following velocity profile,

$u(r)=F(r)-F(\alpha R)+\frac{2 \varepsilon \lambda^{2}}{\eta^{3}} G(r)+u(\alpha R)$

with,

$$
\begin{aligned}
F(r)= & \frac{I_{0}(\kappa R)\left\{4 \zeta_{i} \epsilon \kappa^{2} E_{z} K_{0}(\kappa r)+\kappa\left[\kappa p_{, z}\left(r^{2}-2 \beta^{2} R^{2} \ln (r)\right) K_{0}(\alpha \kappa R)+4 \zeta_{i} \epsilon \kappa^{2} E_{z} \beta R K_{1}(\beta \kappa R) \ln (r)\right]\right\}}{4 \eta \kappa^{2}\left[I_{0}(\kappa R) K_{0}(\alpha \kappa R)-I_{0}(\alpha \kappa R) K_{0}(\kappa R)\right]} \\
& -\frac{I_{0}(\alpha \kappa R)\left\{4 \zeta_{0} \epsilon \kappa^{2} E_{z} K_{0}(\kappa r)+\kappa\left[\kappa p_{, z}\left(r^{2}-2 \beta^{2} R^{2} \ln (r)\right) K_{0}(\kappa R)+4 \zeta_{0} \epsilon \kappa^{2} E_{z} \beta R K_{1}(\beta \kappa R) \ln (r)\right]\right\}}{4 \eta \kappa^{2}\left[I_{0}(\kappa R) K_{0}(\alpha \kappa R)-I_{0}(\alpha \kappa R) K_{0}(\kappa R)\right]} \\
& -\frac{\epsilon \kappa^{2} E_{z}\left[\zeta_{i} K_{0}(\kappa R)-\zeta_{0} K_{0}(\alpha \kappa R)\right]\left\{-4+4 I_{0}(\kappa r)-2(\beta \kappa R)^{2}{ }_{0} F_{1}\left[2,\left(\frac{\beta \kappa R}{2}\right)^{2}\right] \ln (r)\right\}}{4 \eta \kappa^{2}\left[I_{0}(\kappa R) K_{0}(\alpha \kappa R)-I_{0}(\alpha \kappa R) K_{0}(\kappa R)\right]}
\end{aligned}
$$

The slip boundary conditions (Eq. (5)) applied to the inner and outer cylinders are,

$$
u(\alpha R)=\mathcal{L}_{i} \tau_{r z}(\alpha R)
$$$$
u(R)=-\mathcal{L}_{0} \tau_{r z}(R)
$$

where the shear stress distribution $\tau_{r z}(r)$ is given by Eqs. (9) and (11) which can be combined in the form, and $G(r)$ is given by

$G(r)=\int_{\alpha R}^{r} \tau_{r z}^{3} \mathrm{~d} r$

which is an integral function that depends on the radial variation of the shear stress. 


\subsubsection{Exponential PTT model}

For the Exponential viscoelastic model, the integration of Eq (16) results in the following velocity profile,

$u(r)=\int_{\alpha R}^{r} \frac{\tau_{r z}}{\eta} \exp \left(\frac{2 \varepsilon \lambda^{2}}{\eta^{2}} \tau_{r z}^{2}\right) \mathrm{d} r+u(\alpha R)$

which contains an integral that must be solved numerically.

\subsubsection{Dimensionless solutions}

For ease of understanding the above two velocity profiles are written below in a dimensionless form. For the Linear PTT model this is given by,

$$
\begin{aligned}
\frac{u(\bar{r})}{u_{s h}}=\bar{u}(\bar{r})=\bar{F}(\bar{r})-\bar{F}(\alpha \bar{R})+\frac{2 \varepsilon D e^{2}}{\bar{\kappa}^{2}} \bar{G}(\bar{r})+\bar{u}(\alpha \bar{R}) \\
\bar{F}(\bar{r})=\frac{-I_{0}(\bar{\kappa} \bar{R})\left[4 K_{0}(\bar{\kappa} \bar{r})+\Gamma\left(2 \beta^{2} \bar{R}^{2} \ln (\bar{r})-\bar{r}^{2}\right) K_{0}(\alpha \bar{\kappa} \bar{R})+4 \bar{\kappa} \beta \bar{R} K_{1}(\beta \bar{\kappa} \bar{R}) \ln (\bar{r})\right]}{4\left(I_{0}(\bar{\kappa} \bar{R}) K_{0}(\alpha \bar{\kappa} \bar{R})-I_{0}(\alpha \bar{\kappa} \bar{R}) K_{0}(\bar{\kappa} \bar{R})\right)} \\
+\frac{I_{0}(\alpha \bar{\kappa} \bar{R})\left[4 R_{\zeta} K_{0}(\bar{\kappa} \bar{r})+\Gamma\left(2 \beta^{2} \bar{R}^{2} \ln (\bar{r})-\bar{r}^{2}\right) K_{0}(\bar{\kappa} \bar{R})+4 R_{\zeta} \bar{\kappa} \beta \bar{R} K_{1}(\beta \bar{\kappa} \bar{R}) \ln (\bar{r})\right]}{4\left(I_{0}(\bar{\kappa} \bar{R}) K_{0}(\alpha \bar{\kappa} \bar{R})-I_{0}(\alpha \bar{\kappa} \bar{R}) K_{0}(\bar{\kappa} \bar{R})\right)} \\
+\frac{\left[K_{0}(\bar{\kappa} \bar{R})-R_{\zeta} K_{0}(\alpha \bar{\kappa} \bar{R})\right]\left\{-4+4 I_{0}(\bar{\kappa} \bar{r})-2(\beta \bar{\kappa} \bar{R})^{2}{ }_{0} F_{1}\left[2,\left(\frac{\beta \bar{k} \bar{R}}{2}\right)^{2}\right] \ln (\bar{r})\right\}}{4\left[I_{0}(\bar{\kappa} \bar{R}) K_{0}(\alpha \bar{\kappa} \bar{R})-I_{0}(\alpha \bar{\kappa} \bar{R}) K_{0}(\bar{\kappa} \bar{R})\right]}(24)
\end{aligned}
$$

where

$$
\bar{G}(\bar{r})=\int_{\alpha \bar{R}}^{\bar{r}} \bar{\tau}_{r z}^{3} d \bar{r}
$$

For the Exponential PTT model the dimensionless velocity profile is given as,

$$
\bar{u}(\bar{r})=\int_{\alpha \bar{R}}^{\bar{r}} \bar{\tau}_{r z} \exp \left(\frac{2 \varepsilon D e_{\kappa}^{2}}{\bar{\kappa}^{2}} \bar{\tau}_{r z}^{2}\right) \mathrm{d} \bar{r}+\bar{u}(\alpha \bar{R})
$$

where $\bar{u}(\alpha \bar{R})=\overline{\mathcal{L}}_{i} \bar{\tau}_{r z}(\alpha \bar{R})$ (with the dimensionless slip coefficient $\overline{\mathcal{L}}_{i}=\mathcal{L}_{i} \eta / \delta$ and the dimensionless shear stress $\left.\bar{\tau}_{r z}=\tau_{r z} /\left(\eta u_{s h} / \delta\right)\right)$, $D e_{\kappa}=\frac{\lambda u_{s h}}{\xi}=\lambda \kappa u_{s h}$ is the Deborah number based on the EDL thickness and on the Helmholtz-Smoluchowski electro-osmotic velocity, $u_{s h}=-\frac{\epsilon_{\zeta} E_{z}}{\eta}$, the parameter $\Gamma=-\frac{\delta^{2}}{\epsilon_{\zeta}} \frac{p_{z}}{E_{z}}$ represents the ratio of pressure to electro-osmotic driving forces, $R_{\zeta}=\zeta_{0} / \zeta_{i}$ denotes the ratio of the zeta potentials of the two walls, $\bar{\kappa}=\kappa \delta, \bar{R}=\frac{R}{\delta}$ and $\bar{r}=\frac{r}{\delta}$. Note also that $\bar{R}=\frac{1}{1-\alpha}$. For both solutions, the normalized shear stress is given by the same general expression as follows

$$
\begin{aligned}
\bar{\tau}_{r z}(\bar{r})= & \frac{\tau_{r z}}{\eta u_{s h} / \delta}=\frac{\left[\bar{r}-\frac{(\beta \bar{R})^{2}}{\bar{r}}\right] \Gamma}{2}-\bar{\kappa} \frac{-I_{0}(\bar{\kappa} \bar{R})+R_{\zeta} I_{0}(\bar{\kappa} \alpha \bar{R})}{I_{0}(\bar{\kappa} \bar{R}) K_{0}(\alpha \bar{\kappa} \bar{R})-I_{0}(\alpha \bar{\kappa} \bar{R}) K_{0}(\bar{\kappa} \bar{R})} \\
& \times\left[K_{1}(\bar{\kappa} \bar{r})-\frac{\beta \bar{R}}{\bar{r}} K_{1}(\bar{\kappa} \beta \bar{R})\right]-\frac{\bar{\kappa}^{2}}{2} \frac{-K_{0}(\bar{\kappa} \bar{R})+R_{\zeta} K_{0}(\bar{\kappa} \alpha \bar{R})}{I_{0}(\bar{\kappa} \bar{R}) K_{0}(\alpha \bar{\kappa} \bar{R})-I_{0}(\alpha \bar{\kappa} \bar{R}) K_{0}(\bar{\kappa} \bar{R})} \\
& \times\left\{\bar{r}_{0} F_{1}\left[2,\left(\frac{\bar{\kappa} \bar{r}}{2}\right)^{2}\right]-\left[\frac{(\beta \bar{R})^{2}}{\bar{r}}\right]{ }_{0} F_{1}\left[2,\left(\frac{\bar{\kappa} \beta \bar{R}}{2}\right)^{2}\right]\right\}
\end{aligned}
$$

\subsubsection{Determination of $\beta$}

With the help of the slip boundary condition at the outer cylinder, $\bar{u}(\bar{R})=-\overline{\mathcal{L}}_{0} \bar{\tau}_{r z}(\bar{R})$ where $\overline{\mathcal{L}}_{0}=\mathcal{L}_{0} \eta / \delta$, we can obtain $\beta$ by solving,

$H_{l}(\beta) \equiv \bar{F}(\bar{R})-\bar{F}(\alpha \bar{R})+\frac{2 \varepsilon D e_{\kappa}^{2}}{\bar{\kappa}^{2}} \bar{G}(\bar{R})+\bar{u}(\alpha \bar{R})-\bar{u}(\bar{R})=0$

and

$H_{e}(\beta) \equiv \int_{\alpha \bar{R}}^{\bar{R}} \bar{\tau}_{r z} \exp \left(\frac{2 \varepsilon D e_{\kappa}^{2}}{\bar{\kappa}^{2}} \bar{\tau}_{r z}^{2}\right) \mathrm{d} \bar{r}+\bar{u}(\alpha \bar{R})-\bar{u}(\bar{R})=0$

for the Linear and Exponential PTT models, respectively.
To solve numerically Eqs. (28) and (29) we search for a root of $\beta$ in the range $] \alpha, 1$. This can be performed by using an iterative procedure to find the numerical solution of the transcendental Eqs. (28) and (29). Once $\beta$ is known the dimensionless velocity profiles can be obtained from Eqs. (20) and (23) for the Linear and Exponential PTT models, respectively, by numerically solving the integrals in Eqs. (25) and (26). Depending on the dimensionless parameters, one or multiple $\beta$ values can be found. However, in all cases the same velocity and shear stress profiles are obtained with any $\beta$ selected.

\section{Results and discussion}

The solutions obtained in the previous section will now be analyzed first in the absence of slip and then in the presence of slip.

\subsection{No-slip velocity at walls}

In Figs. 2 and 3 we show dimensionless velocity profiles for the pure electro-osmotic flow case $(\Gamma=0)$. As expected, the increase in $\varepsilon D e_{\kappa}^{2}$ leads to an increase in the bulk velocity, due to the increase in the shear thinning leading to an increase in fluidity. The Exponential PTT model shows a higher flow rate when compared to the Linear model since the exponential function promotes a more intense shear thinning for the same shear rate. The difference between the two models also increases with $\varepsilon D e_{\kappa}^{2}$, with both models approaching a Newtonian behavior at low $\varepsilon D e_{\kappa}^{2}$.

For small $\bar{\kappa}$ a quasi-parabolic velocity profile is observed (see Fig. 3), while for high $\bar{\kappa}$ a plug like profile is obtained (see Fig. 2). For small $\bar{\kappa}$ the electric double layer thickness becomes of the order of magnitude of the cylinder gap $(\delta)$ and the region of excess charge is distributed over the entire gap. For high $\bar{\kappa}$ the Debye layer is confined to a small region near the walls, resulting in a plug like flow (Fig. 2).

In terms of the velocity profile skewness, Figs. 2 and 3 show that slightly higher velocities are obtained near the inner cylinder. This happens because of the restrictions imposed on the flow by the wall tangent stresses. Since the outer cylinder has a larger radius, the curvature effect is smaller when compared to the inner cylinder (smaller radius), resulting in slightly smaller velocities.

Fig. 2 also shows that viscoelasticity influences the level of skewness. In order to illustrate the influence of $\varepsilon D e_{\kappa}^{2}$ on the radial location of the null shear stress, $\beta$, Fig. 4 plots the variation of $\beta$ with $\varepsilon D e_{\kappa}^{2}$ for the Linear and Exponential PTT models for $\bar{\kappa}=20$, $\Gamma=0, R_{\zeta}=1$ and $\alpha=0.5$. We found that the value of $\beta$ decreases with the increase in $\varepsilon D e_{\kappa}^{2}$, meaning that the maximum velocity location is moving toward the inner cylinder. This happens because

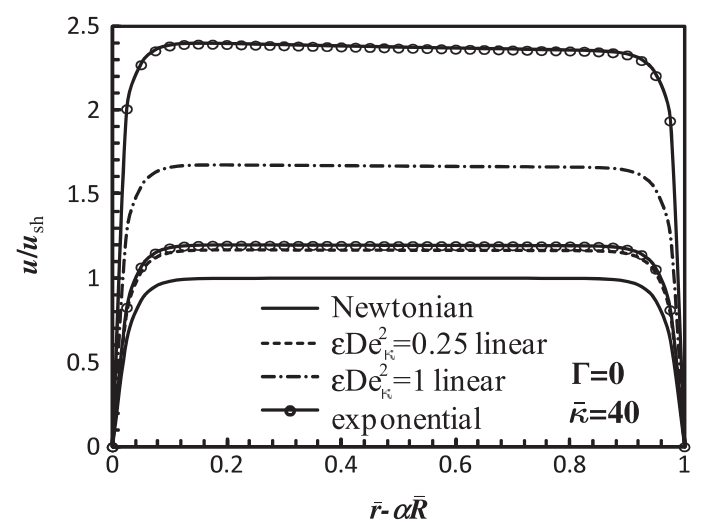

Fig. 2. Velocity profiles for the pure electro-osmotic flow of the Linear and Exponential PTT fluids for $\varepsilon D e_{\kappa}^{2}=\{0,0.25,1\}, \bar{\kappa}=40, \alpha=0.5, R_{\zeta}=1$ and $\overline{\mathcal{L}}_{i}=\overline{\mathcal{L}}_{o}=0$. 


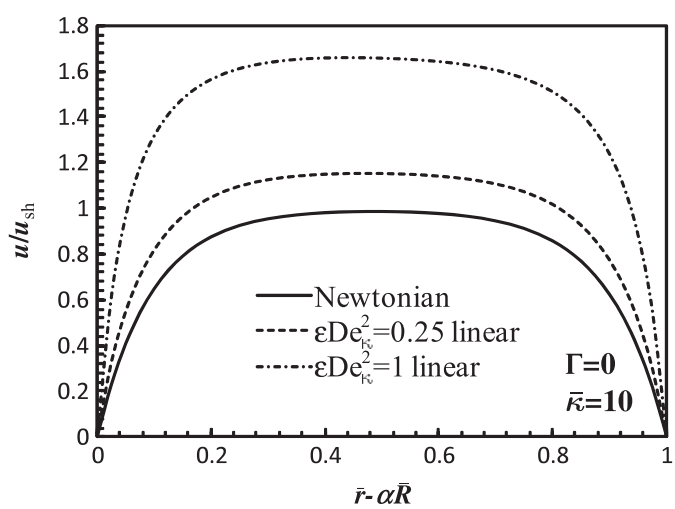

Fig. 3. Velocity profiles for the pure electro-osmotic flow of the Linear PTT model for $\varepsilon D e_{\kappa}^{2}=\{0,0.25,1\}, \bar{\kappa}=10, \alpha=0.5, R_{\zeta}=1$ and $\overline{\mathcal{L}}_{i}=\overline{\mathcal{L}}_{o}=0$.

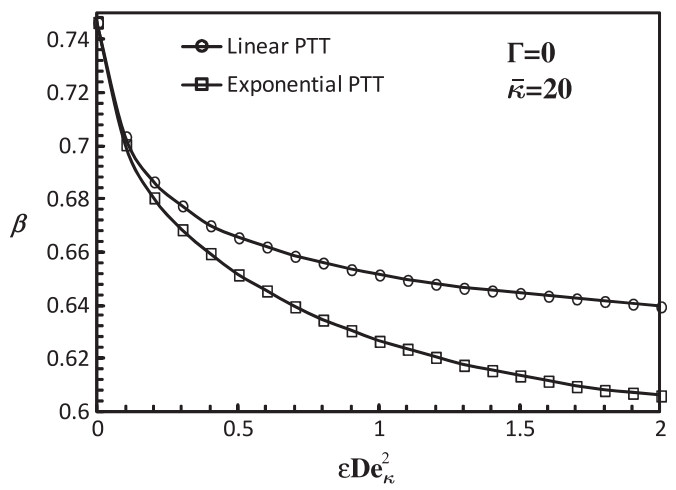

Fig. 4. Variation of $\beta$ with $\varepsilon D e_{\kappa}^{2}$ for the Linear and Exponential PTT models for $R_{\zeta}=1, \bar{\kappa}=20, \Gamma=0, \alpha=0.5$ and $\overline{\mathcal{L}}_{i}=\overline{\mathcal{L}}_{o}=0$.

as $\varepsilon D e_{\kappa}^{2}$ increases, we obtain a stronger shear thinning behavior that enhances the friction force unbalance between the inner and outer cylinders. Also, since the exponential PTT model leads to a stronger shear thinning effect, we obtain smaller values of $\beta$, when compared with the linear PTT model (see Fig. 4). In order to investigate the mixed influence of pressure and electro-osmotic forcings we used two different values for the dimensionless parameter $\Gamma$. For $\Gamma=1$ (adverse pressure gradient) Fig. 5 shows that near the walls the electric potential forcing prevails while far from the walls the adverse pressure gradient effect is visible. The Exponential PTT model shows again a higher bulk velocity, because the stronger shear thinning effect results in a higher fluidity.

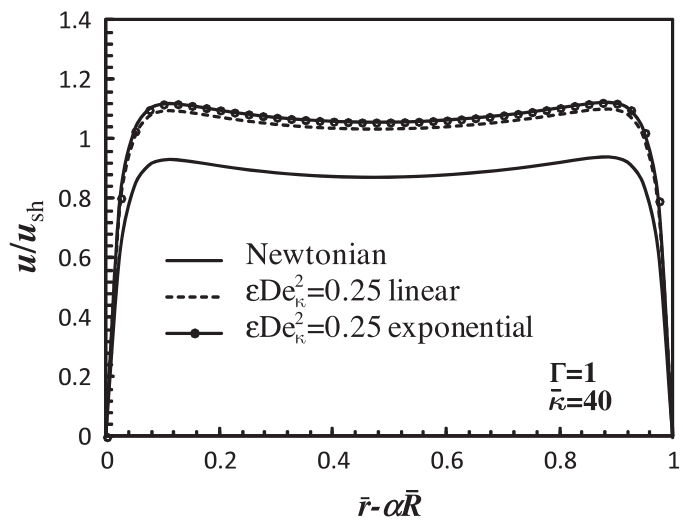

Fig. 5. Velocity profiles for the mixed pressure-electro-osmotic driven flow of the Linear and Exponential PTT models for $\varepsilon D e_{\kappa}^{2}=\{0,0.25\}, \bar{\kappa}=40, \alpha=0.5, R_{\zeta}=1$, $\overline{\mathcal{L}}_{i}=\overline{\mathcal{L}}_{0}=0$ and an adverse pressure gradient, $\Gamma=1$.

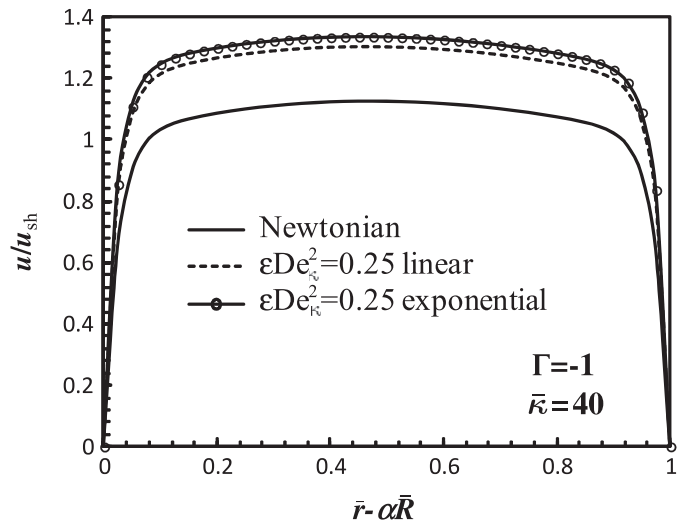

Fig. 6. Velocity profiles for the mixed pressure-electro-osmotic driven flow of the Linear and Exponential PTT models for $\varepsilon D e_{\kappa}^{2}=\{0,0.25\}, \bar{\kappa}=40$ $\alpha=0.5, R_{\zeta}=1, \overline{\mathcal{L}}_{i}=\overline{\mathcal{L}}_{o}=0$ and a favorable pressure gradient, $\Gamma=-1$.

This non-monotonic velocity profile results in three different radial positions $(r)$ where the velocity gradient, $\frac{\mathrm{d} u}{\mathrm{~d} r}$, is null, and, because of that, three solutions of Eq. (28) are possible (Eq. (12) shows that $\tau_{r z}=0$ when $\left.\frac{d u}{d r}=0\right)$. Any of these three solutions for $\beta$ can be used leading to the same solution for the velocity profile.

For $\Gamma=-1$ (Fig. 6) we only have one location of null velocity gradient and the velocity profile is again qualitatively similar to a pressure driven parabolic profile because both pressure gradient and the electro-osmotic forcing are favorable contributions to the flow.

Fig. 7 shows the variation of the normalized flow rate $(\bar{Q})$ with $\Gamma$ for $\varepsilon D e_{\kappa}^{2}=0.5$ and 1 . The dimensionless flow rate was computed numerically with high accuracy using Simpson's 3/8 method and $10^{4}$ equidistant radial intervals. The flow rate is calculated using $\bar{Q}=\left(1 /\left[\bar{R}^{2}\left(1-\alpha^{2}\right)\right]\right) \int_{\alpha \bar{R}}^{\bar{R}} 2 \bar{r} \bar{u}(\bar{r}) \mathrm{d} \bar{r}$. As expected, the normalized flow rate decreases with the increase in $\Gamma$ (i.e. moving from a favorable to an adverse pressure gradient), with smaller flow rate obtained for $\varepsilon D e_{\kappa}^{2}=0.5$ (smaller fluidity when compared to $\varepsilon D e_{\kappa}^{2}=1$, because of the less intense shear thinning). Note that zero net flow occurs for a specific value of the adverse pressure gradient $(\Gamma>0$, not shown) when it compensates the flow driven by electro-osmosis.

\subsection{Slip velocity at walls}

When there is slip at the walls the flow rate driven by electroosmosis increases significantly with the slip coefficient (see Fig. 8). Since the shear stress is different at the inner and outer walls, for the same slip coefficient and $R_{\zeta}=1$ we obtain a higher slip velocity

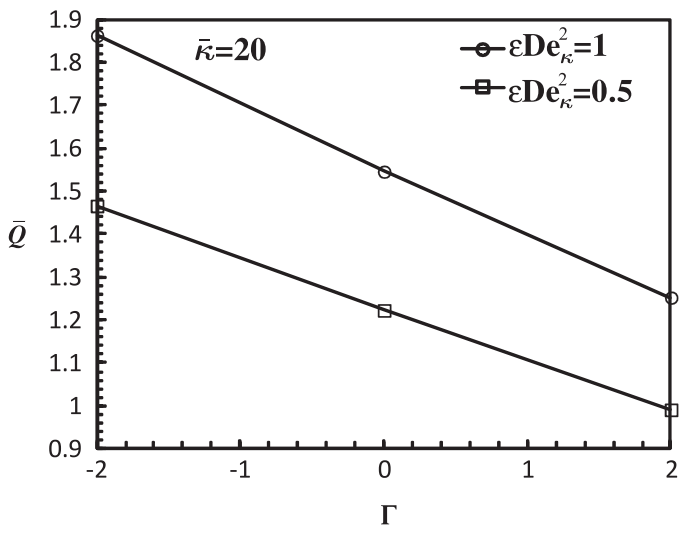

Fig. 7. Variation of the normalized flow rate $(\bar{Q})$ with the dimensionless parameter $\Gamma$ for the Linear PTT model at $\varepsilon D e_{\kappa}^{2}=\{0.5,1\}, \bar{\kappa}=20, \alpha=0.5, R_{\zeta}=1$ and $\overline{\mathcal{L}}_{i}=\overline{\mathcal{L}}_{o}=0$. 


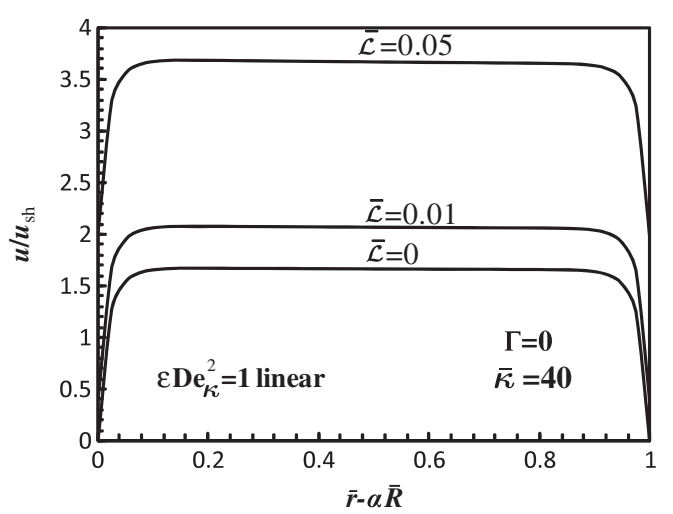

Fig. 8. Effect of the slip velocity coefficient $\overline{\mathcal{L}}=\frac{\mathcal{L} \eta}{\delta}\left(\overline{\mathcal{L}}_{i}=\overline{\mathcal{L}}_{o}=\overline{\mathcal{L}}\right)$ on the velocity profiles for $\varepsilon D e_{\kappa}^{2}=1, \bar{\kappa}=40, \alpha=0.5, R_{\zeta}=1$ and $\Gamma=0$ (Linear PTT model).

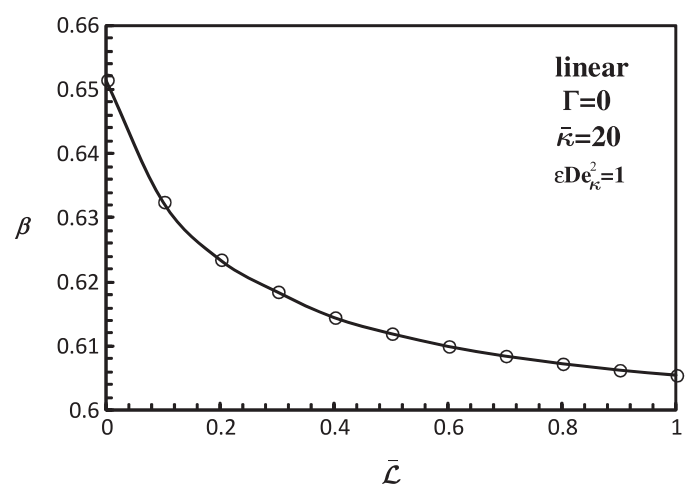

Fig. 9. Variation of $\beta$ with the normalized slip velocity coefficient $\left(\overline{\mathcal{L}}=\frac{\mathcal{L} \eta}{\delta}, \overline{\mathcal{L}}_{i}=\overline{\mathcal{L}}_{0}=\overline{\mathcal{L}}\right)$ for the Linear PTT model $\left(\varepsilon D e_{\kappa}^{2}=1, \bar{\kappa}=20, \alpha=0.5, R_{\zeta}=1\right.$ and $\Gamma=0$ ).

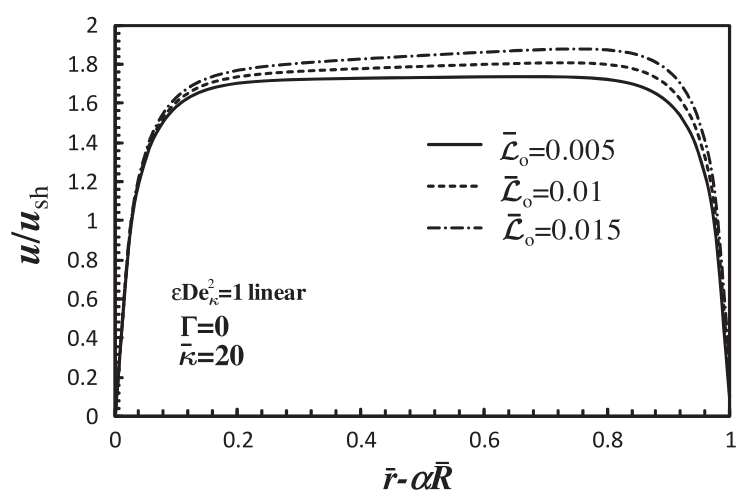

Fig. 10. Effect of different slip velocities at the inner and outer walls on the velocity profile $\left(\overline{\mathcal{L}}_{i}=0, \overline{\mathcal{L}}_{0}\right.$ variable), for $\varepsilon D e_{\kappa}^{2}=1, \bar{\kappa}=20, \alpha=0.5, R_{\zeta}=1$ and $\Gamma=0$ (Linear PTT model).

at the inner wall than at the outer wall, but still the skewness of the velocity profiles shown is very small.

Fig. 9 plots the variation of $\beta$ with the slip coefficient $\overline{\mathcal{L}}$ for a constant $\varepsilon D e_{\kappa}^{2}=1$, showing that $\beta$ decreases with $\overline{\mathcal{L}}$. This means that increasing the slip velocity moves the maximum velocity location toward the inner cylinder, akin to what happened with the increase in $\varepsilon D e_{\kappa}^{2}$ for the no-slip case. This happens because the maximum velocity moves toward the less restrictive wall which in this case is the inner cylinder. As a simple example, we can imagine the flow between parallel plates, with full slip at one of the walls. The velocity profile would then be a half parabola, with maximum velocity at the slippery wall. The local wall shear stress is higher at the inner cylinder (for the no-slip case), and, based on the slip velocity model, Eq. (5), this promotes a higher slip velocity, therefore, a less restrive inner wall.

We also analyzed different velocity profiles assuming different slip velocities at the inner and outer cylinders. For ease of understanding we assume a no-slip velocity at the inner wall and different slip velocities at the outer wall (Fig. 10). As shown in Fig. 10, an increase in the flow rate is obtained, due to the friction decrease at the outer wall. We can also observe that for a small slip coefficient a more symmetric velocity profile is obtained, since the presence of slip velocity at the outer cylinder decreases the shear force to values similar to the ones obtained at the inner cylinder wall.

\section{Conclusions}

Semi-analytical solutions for the pressure driven/electro-osmotic annular flow of viscoelastic fluids modeled by the Linear and Exponential PTT models were presented with and without the assumption of slip velocity at the walls. We found that for a constant pressure gradient the Exponential PTT model shows a higher flow rate when compared to the Linear model. The presence of slip velocity leads to an increase in the flow rate and to an intensified skewness of the velocity profiles.

\section{Acknowledgements}

The authors gratefully acknowledge funding by COMPETE, FEDER and Fundação para a Ciência e a Tecnologia (FCT) through Projects PEst-C/CTM/LA0025/2013 (Strategic Project - LA 252013-2014), PTDC/EQU-FTT/113811/2009 and PDTC/EME-MFE/ $113988 / 2009$. AMA would also like to thank FCT for financial support through the scholarship SFRH/BPD/75436/2010.

\section{References}

[1] M.M. Denn, Annu. Rev. Fluid Mech. 33 (2001) 265-287.

[2] E. Lauga, M.P. Brenner, H.A. Stone, in: C. Tropea, A. Yarin, J.F. Foss (Eds.), Handbook of Experimental Fluid Dynamics (Chapter 19), Springer, 2007, pp. 1219-1240.

[3] I.B. Kazatchkov, S.G. Hatzikiriakos, Rheol. Acta 49 (2010) 267-274.

[4] S.G. Hatzikiriakos, Prog. Polym. Sci. 37 (2012) 624-643.

[5] C.L.M.H. Navier, Mem. Acad. R. Sci. Inst. Fr. 6 (1827) 389-440.

[6] Y. Zhu, S. Granick, Phys. Rev. Lett. 87 (2001) 096105.

[7] D.C. Tretheway, C.D. Meinhart, Phys. Fluids 14 (2002) L9-L12.

[8] D.C. Tretheway, C.D. Meinhart, Phys. Fluids 16 (2004) 1509-1515.

[9] V. Tandon, B.J. Kirby, Electrophoresis 29 (2008) 1102-1114.

[10] H.M. Park, T.W. Kim, Lab. Chip. 9 (2009) 291-296.

[11] C.Y. Soong, P.W. Hwang, J.C. Wang, Microfluid. Nanofluid. 9 (2010) 211-223.

[12] G.D. Ngoma, F. Erchiqui, Int. J. Therm. Sci. 46 (2007) 1076-1083.

[13] J. Jamaati, H. Niazmand, M. Renksizbulut, Int. J. Therm. Sci 49 (2010) 11651174.

[14] C. Zhao, C. Yang, Biomicrofluidics 5 (2011) 014110.

[15] A.M. Afonso, M.A. Alves, F.T. Pinho, J. Non-Newt. Fluid Mech. 159 (2009) 5063.

[16] A.M. Afonso, M.A. Alves, F.T. Pinho, J. Eng. Math. 71 (2011) 15-30.

[17] M.P. Escudier, P.J. Oliveira, F.T. Pinho, Int. J. Heat Fluid Flow 23 (2002) 52-73.

[18] M.P. Escudier, P.J. Oliveira, F.T. Pinho, S. Smith, Exp. Fluids 33 (2002) 101-111.

[19] R.K. Bhatnagar, J. Ind. Inst. Sci. 45 (1963) 126-151.

[20] F.T. Pinho, P.J. Oliveira, J. Non-Newt. Fluid Mech. 93 (2000) 325-337.

[21] D.O. Cruz, F.T. Pinho, J. Non-Newt. Fluid Mech. 121 (2004) 1-14.

[22] S. Wang, M. Xu, Non. Anal.: Real World Applications 10 (2009) 1087-1096.

[23] H.-K. Tsao, Journal of Colloid and Interface Science 225 (2000) 247-250.

[24] Y. Kang, C. Yang, X. Huang, Journal of Colloid and Interface Science 253 (2002) $285-294$.

[25] P. Goswami, S. Chakraborty, Microfluid Nanofluid 11 (2011) 255-267.

[26] Y. Jian, L. Yang, Q. Liu, Phys Fluids 22 (2010) 042001.

[27] M. Shamshiri, R. Khazaeli, M. Ashrafizaadeh, S. Mortazavi, J. Non-Newtonian Fluid Mech. 173-174 (2012) 1-12.

[28] N. Phan-Thien, R.I. Tanner, J. Non-Newtonian Fluid Mech. 2 (1977) 353-365.

[29] N. Phan-Thien, J. Non-Newtonian Fluid Mech. 26 (1988) 327-340.

[30] J. Dutka, Arch. History Exact Sci. 31 (1984) 15-34.

[31] A. Cuyt, V.B. Petersen, B. Verdonk, H. Waadeland, W.B. Jones, Handbook of continued fractions for special functions, Springer, 2008. Chapter 16. 